\title{
Effect of lentivirus-mediated shRNA targeting VEGFR-3 on proliferation, apoptosis and invasion of gastric cancer cells
}

\author{
XIAN-JU QIN, DE-JIAN DAI, ZHI-GUANG GAO, JIN-LIANG HUAN and LING ZHU \\ Department of General Surgery, Shanghai Eighth People's Hospital, Shanghai, P.R. China
}

Received May 10, 2011; Accepted June 14, 2011

DOI: $10.3892 / \mathrm{ijmm} .2011 .758$

\begin{abstract}
It has been reported that vascular endothelial growth factor receptor 3 (VEGFR-3) is highly expressed in most tumor tissues, including gastric cancer. However, the effects of VEGFR-3 knockdown on the proliferation, apoptosis and invasion of gastric cancer cells and downstream signaling molecules have not yet been well established. In the present study, four short hairpin RNA (shRNA) sequences targeting the VEGFR-3 gene (NM_002020) were designed and cloned into a lentiviral vector, pRNAT-U6.2/Lenti, to construct four recombinant lentiviral vectors. The vectors with the two highest interfering efficiencies were selected to be co-transfected with packaging vectors in HEK293T cells to assemble lentivirus particles. Results from Western blot analysis showed that the VEGFR-3 shRNA-4 lentivirus-infected group (sh\#4) had the highest efficiency of gene silencing in the MKN45 cell line compared with the parental and control group. The sh\#4 group significantly slowed cell proliferation, decreased the mean percentage of S-phase cells and increased the mean percentage of G1 phase cells, promoted cell apoptosis, and also significantly inhibited cell invasion of MKN45 compared with the other two groups. Furthermore, the expression of the anti-apoptotic factor Bcl-2 was significantly decreased in the sh\#4 group compared to that of the other two groups. Moreover, results from qRT-PCR revealed that knockdown of VEGFR-3 with the shRNA lentiviral vector resulted in down-regulation of the downstream neural cell adhesion molecule contactin-1 (CNTN-1). In conclusion, the recombinant lentivirus particles were able to remarkably suppress VEGFR-3 expression, regulate the cell cycle, inhibit proliferation and induce apoptosis in the MKN45 cell lines.
\end{abstract}

\section{Introduction}

Gastric cancer is the second most common cause of cancerrelated death in males and the fourth in females. Despite

Correspondence to: Dr Xian-ju Qin, Department of General Surgery, Shanghai Eighth People's Hospital, 8 Caobao Road, Shanghai 200235, P.R. China

E-mail: qinxianju@126.com

Key words: lentivirus, short hairpin RNA, VEGFR-3, contactin-1, gastric cancer its decreasing incidence, it remains a common malignancy worldwide. The biologic heterogeneity of neoplasms is the principal cause of the failure of antitumor therapy. Targeting the neovasculature of tumors has been explored as a way of attacking more essential components of the tumors (1). Better understanding of the biology of gastric cancer and development of new approaches to therapy of gastric cancer are critical.

Growth factors and their receptors play a pivotal role in the regulation of cancer progression and neovascularization (2). In particular, vascular endothelial growth factor receptors (VEGFRs) have been extensively investigated as targets for antineoplastic therapy. The VEGFRs are a subfamily of receptor tyrosine kinases that play key roles in angiogenesis and lymphangiogenesis $(3,4)$. It is well known that VEGF acts by binding to two high affinity receptor tyrosine kinases: VEGF receptor (VEGFR-1), also called Flt-1, and VEGFR-2, also called Flk-1/KDR. Although the role of VEGFR-1 in VEGF signaling is still debated, VEGFR-2 is effective in transducing signals regulating cell division and inhibition of cell death.

Presence of VEGFR-3 in tumor cells has been proposed to be a possible predictive factor to determine the clinical approach because it has been closely related with lymph node metastasis or poor prognosis in patients (5-9). Recent studies have demonstrated that VEGFR-3, also named Flt-4, is not only expressed in endothelial cells but also in a wide variety of malignant tumors, including gastric cancer $(7,10)$, colorectal and breast cancers (11), prostate cancer (12), and non-small cell lung carcinoma (5). The VEGF-C/VEGFR-3 axis has been studied extensively in the tumor process, including neovascularization (4,11,13-16), lymph node metastasis $(6,8,17-19)$, tumor growth, invasion and metastasis $(12,15,20-25)$. For example, previous studies have demonstrated that the VEGF-C/VEGFR-3 axis promotes invasion and metastasis of lung adenocarcinoma cells $(5,20)$, progression of prostate cancer (12) and proliferative and antiapoptotic activity in leukemia cells (26) and mesothelioma cells (27). Blockade of the VEGF-C/VEGFR-3 axis inhibits tumor neovascularization, lymph node metastasis and tumor progression $(9,19,22,25)$. Targeting the VEGF/VEGFR signaling has been used for the treatment of solid tumors. Several experimental approaches with either a VEGFR tyrosine kinase inhibitor that mimics an antibody against VEGF $(9,28,29)$ or targeting of VEGFR by RNAi technology $(14,19)$ have demonstrated in inhibition of angiogenesis and tumor progression. All of these studies indicate the promotion effect 
Table I. Sequences of four pairs of short hairpin RNAs (P-1, P-2 and P-3).

Name

Sequences

VEGFR-3 sh\#1 Oligo1: 5'GATCCGAGAATAAGACTGTGAGCAATTCAAGAGATTGCTCACAGTCTTATTCTTTTTTTC3'

Oligo2: 3'GCTCTTATTCTGACACTCGTTAAGTTCTCTAACGAGTGTCAGAATAAGAAAAAAAGAGCT5'

VEGFR-3 sh\#2 Oligo1: 5'GATCCGAGCTCCGACATCAGCGCGTTCAAGAGACGCGCTGATGTCGGAGCTCTTTTTTC3' Oligo2: 3'GCTCGAGGCTGTAGTCGCGCAAGTTCTCTGCGCGACTACAGCCTCGAGAAAAAAGAGCT5'

VEGFR-3 sh\#3 Oligo1:5'GATCCGAAACTCAGCCCACACGGTGTTCAAGAGACACCGTGTGGGCTGAGTTTTTTTTTC3' Oligo2: 3'GCTTTGAGTCGGGTGTGCCACAAGTTCTCTGTGGCACACCCGACTCAAAAAAAAAGAGCT5'

VEGFR-3 sh\#4 Oligo1:5'GATCCGCATCACGGAGGAGTCACACTTCAAGAGAGTGTGACTCCTCCGTGATGTTTTTTC3' Oligo2: 3'GCGTAGTGCCTCCTCAGTGTGAAGTTCTCTCACACTGAGGAGGCACTACAAAAAAGAGCT5'

Each DNA template consists of a 19-nt sense siRNA sequence linked to its reverse complementary antisense siRNA sequence by a 9-nt loop. Sense and antisense strands are indicated in bold.



Figure 1. Schematic diagram of the lentivirus vector and structure of the DNA template. (A) Schematic diagram of the pRNAT-U6.2/Lenti vector. The DNA template was ligated with BamHI and XhoI restriction sites in the downstream U6.2 promoter. Transcripts of the template will produce shRNA which has a 19 nt double strand stem with a 9 nt loop which targets VEGFR-3 mRNA or no specific human gene mRNA. (B) Structure of the DNA template. The length of the palindrome sequence (19 nucleotide sense strand and antisense strand) were separated by a 9 nucleotide spacer followed by four continuous thymines (T) as termination signal. Two restriction enzyme sites (BamHI and XhoI) were added to the 2 termination ends.

of VEGFR-3 in tumor progression, suggesting that blockade of VEGFR tyrosine kinase is an effective antivascular therapy because tumor-associated vessels are specifically targeted.

The work by Su et al identified that VEGFR-3 activated the Src/p38 mitogen-activated protein kinase (MAPK) signaling by up-regulating the expression of CNTN-1 in lung adenocarcinoma cells (5). However, the biological functions of
VEGFR-3 and the downstream neural cell adhesion molecule contactin-1 in human gastric cancer cells has not yet been well established.

We therefore hypothesized that suppression of VEGFR-3 in human gastric cancer cells can inhibit cancer progression. To test this hypothesis, we silenced VEGFR-3, expressed in the cancer cell line MKN45, by lentivirus-mediated shRNA and 
Table II. Real-time quantitative RT-PCR primers.

\begin{tabular}{lll}
\hline Targets & \multicolumn{1}{c}{ Forward primer } & \multicolumn{1}{c}{ Reverse primer } \\
\hline VEGFR-3 & 5'CGGGGAAGGGGAGGGAGGAG3' & 5'GAAAGGCGGCGGGTGTCAGG3 \\
CNTN-1 & 5'TGTTCAGCAAATTCATCCCA3' & 5'TCTACCCACTCAGGGAATGC3' \\
GAPDH & 5'TAAGTATGACTCCACCCACG3' & 5'CTAGCACCTTCCCAACTA3' \\
\hline
\end{tabular}

examined its effects on gastric cancer cell functions, including cell proliferation, cell apoptosis, cell cycle and cell invasion. Furthermore, downstream molecule CNTN-1 was found to be down-regulated with VEGFR-3 knockdown.

\section{Materials and methods}

Construction of VEGFR-3 shRNA recombinant lentiviral vectors and preparation of lentivirus particles. Four potential oligonucleotides sequences that specific target the VEGFR-3 gene mRNA (GenBank accession no. NM_002020) smallinterfering RNAs (siRNAs) were synthesized using the Ambion online siRNA finder (www.ambion.com). A scrambled sequence of the VEGFR-3 target sequences was used as a negative control. Sequences were confirmed by NIH BLAST analysis to have no substantial homology to sequences in other vertebrate genes. shRNA lentiviral vectors were constructed as described in Fig. 1 using the shRNA sequences listed in Table I. Annealed double-stranded oligonucleotides were ligated into endonuclease $\mathrm{BamHI}$ and $\mathrm{XhoI}$ digestion sites of the pRNAT-U6.2/Lenti vector (GenScript, USA) to create the new recombinant plasmids pRNAT-control shRNA (con sh), pRNAT-shRNA1 (sh\#1), pRNAT-shRNA2 (sh\#2), pRNATshRNA3 (sh\#3) and pRNAT-shRNA4 (sh\#4). Finally, they were further verified by DNA sequencing. For preparation of lentivirus particles, $48 \mathrm{~h}$ post co-transfection of the recombinant lentiviral vectors (con sh, sh\#1 and sh\#4) and the lentiviral packaging vectors, the pPACKH1-Lenti vector Packaging kit (SBI, USA) was used in HEK 293T cells. The supernatants were filtered and stored at $-80^{\circ} \mathrm{C}$.

Cell culture. The human gastric cancer cell line MKN45 (Shanghai Institute of Biochemistry and Cell Biology, Chinese Academy of Sciences) was maintained in RPMI-1640, supplemented with $10 \%$ fetal bovine serum (FBS) (Gibco, USA), $100 \mathrm{U} / \mathrm{ml}$ penicillin and $100 \mu \mathrm{g} / \mathrm{ml}$ streptomycin (Gibco). HEK293T cells were maintained in DMEM with 10\% FBS (Gibco). Both cells were incubated at $37^{\circ} \mathrm{C}$ in a mixture of $5 \%$ $\mathrm{CO}_{2}$ and $95 \%$ air.

Transfection of VEGFR-3 shRNA recombinant lentiviral vectors and lentivirus particles infection. Transient transfection of VEGFR-3 shRNA recombinant lentiviral vectors were carried out using Lipofectamine 2000 (Invitrogen, USA). One day before transfection, MKN45 cells were trypsinized and seeded on a 6-well plates at a density of $1 \times 10^{5}$ cells/well in $1 \mathrm{ml}$ of culture medium supplemented with RPMI-1640 and 10\% serum without antibiotics for $24 \mathrm{~h}$. Thereafter, $2 \mu \mathrm{g}$ of plasmid diluted in $50 \mu \mathrm{l}$ of Opti-MEM and $5 \mu \mathrm{l}$ of Lipofectamine 2000 diluted in $50 \mu 1$ of Opti-MEM was preincubated for $5 \mathrm{~min}$. The
2 mixtures were combined and incubated for $25 \mathrm{~min}$ at room temperature for complex formation. After addition of $150 \mu \mathrm{l}$ of Opti-MEM, the entire mixture was added to each well. Cells were assayed 2 days after transfection. The cells were harvested $48 \mathrm{~h}$ after transfection and used for the experiments. For recombinant lentivirus particles infection, non-infected MKN45 cells (parental), VEGFR-3 control shRNA infected (con sh) and VEGFR-3 shRNA infected MKN45 cells (sh\#1 and sh\#4) were used. The MKN45 cells for each group were seeded in a 6-well plate and incubated at $37^{\circ} \mathrm{C}$ in $5 \% \mathrm{CO}_{2}$ until cell confluence reached $30 \%$. Later $2 \times 10^{6}$ transducing units (TU) per well of the recombinant lentivirus were added to the plate and incubated at $37^{\circ} \mathrm{C}$ in $5 \% \mathrm{CO}_{2}$ for 3 days.

Real-time quantitative reverse transcription polymerase chain reaction $(q P C R)$. Total-RNA was isolated using a homogenizer and TRIzol ${ }^{\mathrm{TM}}$ reagent (Invitrogen) according to the manufacturer's instructions. RNA extraction and cDNA synthesis were performed as described previously (30). The quality of RNA in samples was determined by electrophoresis through agarose gels and staining with ethidium bromide; 18S and 28S RNA bands were visualized with UV illumination. The primers used, were based on the cDNA sequence of VEGFR-3 (NM_002020); glyceraldehyde-3-phosphate dehydrogenase (GAPDH) was used as an internal control (Table II). The reaction conditions were: $95^{\circ} \mathrm{C}$ for $10 \mathrm{~min}$; followed by 40 cycles at $95^{\circ} \mathrm{C}$ for $15 \mathrm{sec}, 56^{\circ} \mathrm{C}$ for $30 \mathrm{sec}$ and $72^{\circ} \mathrm{C}$ for $30 \mathrm{sec}$. Real-time PCR amplifications were performed using an ABI PRISM 7000 cycler (Applied Biosystems) and threshold cycle numbers $\left(C_{T}\right)$ were transformed using the $\Delta C_{T}$ comparative method. Each sample was analyzed in triplicate. Gene-specific expression values were normalized to the expression values of GAPDH (endogenous control) within each sample. Relative quantification was performed using the comparative method. The amount of target, normalized to an endogenous control and relative to a calibrator, was determined by the comparative $\mathrm{C}_{\mathrm{T}}$ method $\left(\Delta \Delta \mathrm{C}_{\mathrm{T}}\right)$. In brief, the $\Delta \mathrm{C}_{\mathrm{T}}$ value was determined by subtracting the average GAPDH $\mathrm{C}_{\mathrm{T}}$ from the average target gene value in the same sample. The calculation of $\Delta \Delta \mathrm{C}_{\mathrm{T}}$ involves subtraction of the $\Delta \mathrm{C}_{\mathrm{T}}$ calibrator value.

Protein extraction and Western blot analysis. At 3 days after the infection, cells from the parental, con sh, sh\#1 and sh\#4 lentivirus particles infected group were lysed with RIPA lysis buffer. Cell lysates containing equal amounts of protein were separated on $8 \%$ SDS-PAGE (sodium dodecyl sulfatepolyacrylamide gel electrophoresis) and electrotransferred onto nitrocellulose (NC) membranes (Amersham, Arlington Heights, IL, USA). The membranes were initially blocked with 5\% non-fat dry milk in Tris-buffered saline (TBS) for 

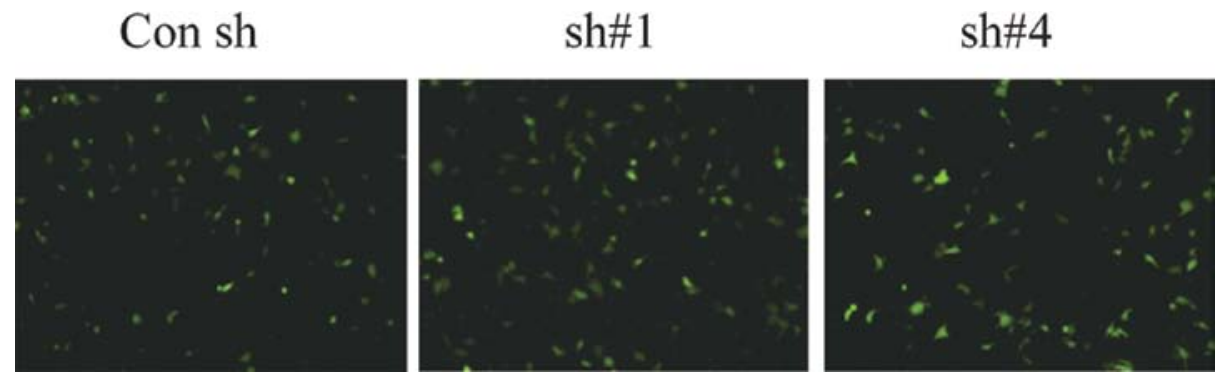

Figure 2. Observation of the transfection efficiency in MKN45 cells under a fluorescence microscope at $72 \mathrm{~h}$ (x200 magnification). Left panel, con sh group, middle, sh\#1 group and the right panel, sh\#4 group.

$1 \mathrm{~h}$ at room temperature and then incubated with the primary antibodies anti-VEGFR-3, anti-Bcl-2 and anti-GAPDH (Santa Cruz Biotechnology; Santa Cruz, CA, USA) at $4^{\circ} \mathrm{C}$ overnight and with the horseradish peroxidase HRP-conjugated secondary antibodies (Jackson ImmunoResearch, PA, USA) for $2 \mathrm{~h}$ at room temperature. Protein bands were then developed with enhanced chemiluminescence (ECL) detection reagents (Amersham Biosciences, Piscataway, NJ, USA). All analyses were performed in duplicate.

MTT assay. Cell proliferation in vitro was analyzed with the tetrazolium salt 3-(4,5-dimethylthiazol-2-yl)-2,5-diphenyltetrazolium bromide (MTT). Cells were seeded in quadruplicates on 96-well plates at a density of $1 \times 10^{4}$ cells/well in $100 \mu \mathrm{l}$ medium, then incubated in a $37^{\circ} \mathrm{C}$ humidified incubator for attachment. The MTT (Sigma, USA) assay was performed according to the manufacturer's manual. Briefly, cells were infected with con sh and sh\#4 lentivirus particles after 24, 48 and $72 \mathrm{~h}$, then $20 \mu \mathrm{l}$ of $5 \mathrm{mg} / \mathrm{ml}$ MTT dissolved in PBS were added to each well, and cells were incubated for another $4 \mathrm{~h}$, followed by the addition of $150 \mu \mathrm{l}$ DMSO and left for $30 \mathrm{~min}$ at room temperature for colour development. Optical density was determined using a $490 \mathrm{~nm}$ filter on a microplate reader (Tecan, Groedig, Austria). The absorbance values were determined at 1,2,3 days in each group. Each assay was performed in triplicate and the experiment was repeated at least on 3 separate occasions. The relative cell proliferation was calculated as A492 $\mathrm{nm}$ of con sh or sh\#4 group/A492 nm of the parental group.

Cell cycle distribution. Three days after the infection, cells from the parental, con sh and sh\#4 lentivirus particles-infected groups were collected by trypsinization and centrifugation, washed twice with cold PBS, and fixed with cold $70 \%$ ethanol at $4^{\circ} \mathrm{C}$ overnight. The fixed cells were collected, washed twice with PBS and suspended in PBS containing $10 \mu \mathrm{g} / \mathrm{ml}$ propidium iodide (PI, Sigma) and $100 \mu \mathrm{g} / \mathrm{ml}$ RNase A, then incubated at $4^{\circ} \mathrm{C}$ for at least $30 \mathrm{~min}$ avoiding light to eliminate the intracellular RNA. The cell cycle distribution was determined using flow cytometric analysis (Becton-Dickinson FACScan).

Transwell assay. The invasion assay was performed using transwell chambers with filter inserts (pore size, $8 \mu \mathrm{m}$ ) coated with Matrigel (40 mg, BD Bioscience, USA) in 24-well dishes (Corning, USA). Approximately $2 \times 10^{4}$ cells from parental,

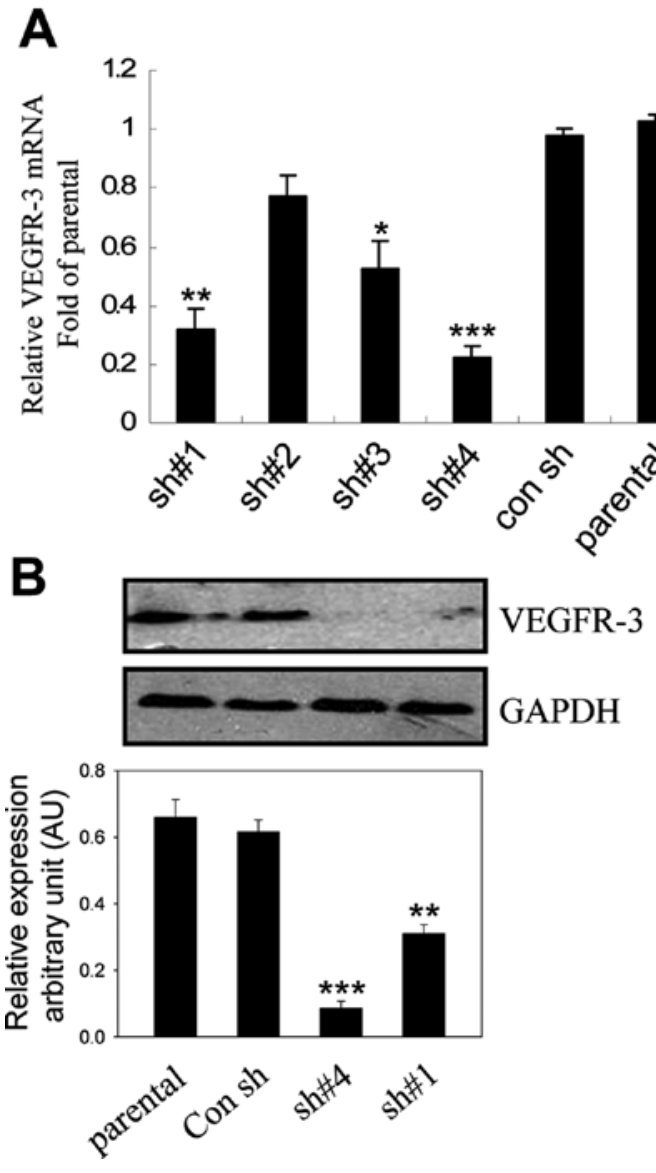

Figure 3. Suppression of VEGFR-3 expression with the lentiviral expression vector in MKN45 cells. (A) The MKN45 cells were transfected with four lentiviral expression vectors (sh\#1, sh\#2, sh\#3 and sh\#4) or a con sh vector using Lipofectamine 2000. The expression of VEGFR-3 mRNA was examined by qPCR. GAPDH served as an internal control. (B) Con sh, sh\#1 and sh\#4 lentivirus expression vectors were packaged into lentivirus particlesinfected MKN45 cells. VEGFR-3 protein levels were analysis by Western blot analysis. Protein quantification was performed by densitometric analysis of the protein band area. The protein product quantified was relative to the internal control GAPDH. All experiments were performed at least 2 times with consistent and repeatable results. ${ }^{*} \mathrm{P}<0.05 ;{ }^{* * *} \mathrm{P}<0.01 ;{ }^{* * *} \mathrm{P}<0.001$.

con sh and the sh\#4 group in $200 \mu 1$ of serum-free RPMI-1640 medium were placed in the upper chamber and $300 \mathrm{ml}$ of the same medium was placed in the lower chamber. The plates were incubated for $24 \mathrm{~h}$ at $37^{\circ} \mathrm{C}$ in $5 \% \mathrm{CO}_{2}$, then cells were fixed in methanol for $15 \mathrm{~min}$ and stained with $0.1 \%$ crystal violet for $15 \mathrm{~min}$. Cells on the upper side of the filters were removed with cotton tipped swabs and the filters were washed 
A

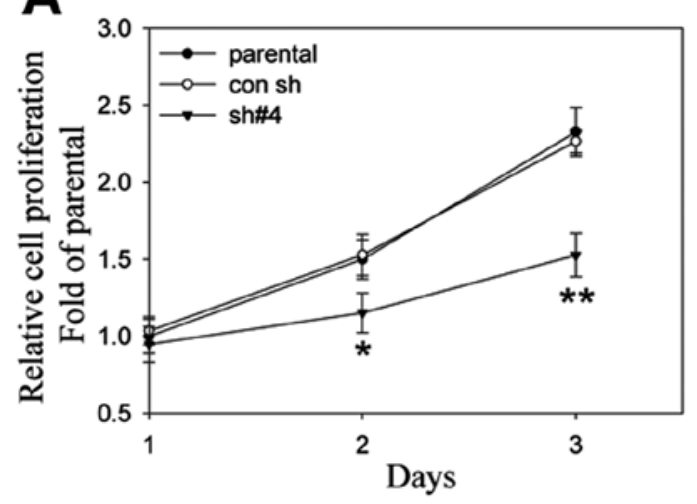

B

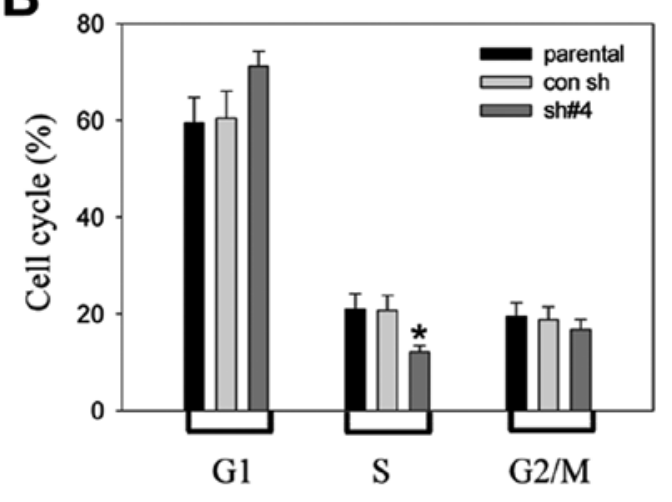

Figure 4. Effect of lentiviral expression vector-mediated inhibition of VEGFR-3 on proliferation and cell cycle stage in MKN45 cell in vitro. (A) MKN45 cells were infected with con sh, sh\#4 lentivirus particles for 24, 48 and $72 \mathrm{~h}$. Cell proliferation was assessed by the MTT assay as described in Materials and methods. (B) Cells were collected $72 \mathrm{~h}$ after infection with con sh, sh\#4 lentivirus particles and their DNA content was subsequently assayed by flow cytometry. ${ }^{*} \mathrm{P}<0.05 ;{ }^{* *} \mathrm{P}<0.01$. All of the experiments were repeated at least three times.

with PBS. Invaded cells in 5 random fields were counted under a light microscope and photographed. Each assay was performed in triplicate and each independent experiment was repeated at least two times. The relative invasiveness rate was calculated as the fold of cells that had successfully invaded through the Matrigel-coated membrane to the lower wells in the con sh and the sh\#4 group relative to the parental group.

Statistical analysis. The results were presented as mean \pm standard deviation (SD). The data were analyzed using one-way analysis of variance (ANOVA) or one-way ANOVA on ranks with multiple comparison followed by Dunnett's tests to determine where differences among groups existed. Statistical significance was accepted when $\mathrm{P}<0.05$.

\section{Results}

Infection efficiency. Seventy-two hours after infection of MKN45 cells, the infection efficiency was detected under fluorescence microscope. A large number of cells illuminated bright green fluorescence, which represented a high infection efficiency (Fig. 2).

qPCR and Western blot analysis assay for the interfering efficiency in gastric cancer cells. First, qPCR was performed to measure the VEGFR-3 mRNA levels from four recombinant lentiviral vectors transfected in the gastric cancer cell line MKN45 using Lipofectamine 2000. The expression of VEGFR-3 mRNA decreased by $\sim 45,65$ and $78 \%$ in the sh\#3, sh\#1 and sh\#4 groups, respectively compared to the con sh group (Fig. 3A). To further confirm this interfering efficiency, the vectors with the two highest interfering efficiencies (sh\#1 and sh\#4) were selected to be co-transfected with packaging vectors in HEK293T cells to assemble lentivirus particles. Western blot analysis was performed to examine the expression levels of VEGFR-3 in the MKN45 cell line infected by lentivirus particles. Results showed that VEGFR-3 levels were significantly reduced by $\sim 50$ and $68 \%$ in the sh\#1 and sh\#4 groups, respectively, compared with the other two groups (Fig. 3B), which indicates that the sh\#4 group had the highest interfering efficiency in MKN45 cells.
VEGFR-3 knockdown inhibition of gastric cancer cell proliferation and cell cycle entry of gastric cancer cells. To determine whether VEGFR-3 knockdown in MKN45 cells affects cell proliferation and cell cycle distribution, we determined the MKN45 cell proliferation and cell cycle stage by the MTT assay and flow cytometry, respectively. At 2 and 3 days after infection, MTT assay results showed that the sh\#4 group, when compared with the parental and con sh groups, exhibited significant inhibition of cancer cell proliferation (Fig. 4A). We further analyzed the DNA contents of cell populations reflecting the cell cycle distribution after down-regulation of VEGFR-3 expression. A slight increase in the G1 phase population with a concomitant remarkable decrease in the $S$ phase population was observed after VEGFR-3 knockdown (Fig. 4B), suggesting that the lentivirus-mediated inhibition of VEGFR-3 could suppress cell proliferation in vitro.

Lentivirus-mediated inhibition of VEGFR-3-induced cell apoptosis in gastric cancer cells. We next addressed if inhibition of VEGFR-3 expression would induce cell apoptosis. Three days after infection, apoptosis was measured with an Annexin V/FITC kit according to the manufacturer's protocol. sh\#4 but not the con sh group significantly increased cell apoptosis in MKN45 cells compared with the parental group (Fig. 5A). The rate of cell apoptosis in VEGFR-3 knockdown cells was $\sim 18 \%$, an almost 3 -fold increase over the rate of the other two groups. To clarify the molecular mechanism of VEGFR-3 knockdown in cell apoptosis, Western blot analysis was employed to evaluate the expression of the anti-apoptotic factor Bcl-2. The expression of Bcl-2 obviously decreased in the sh\#4 group compared to the other two groups (Fig. 5B). These results indicate that the lentivirus-mediated inhibition of VEGFR-3 led to increased cell apoptosis in vitro.

The effect of VEGFR-3 knockdown on the invasion ability of gastric cancer cells. At 3 days after infection, the number of invasive MKN45 cells in the sh\#4 group was significantly lower than that of the other two groups as indicated by fluorescence microscopic examination (Fig. 6). The results indicate that lentivirus-mediated inhibition of VEGFR-3 suppressed the invasive ability of cancer cells in vitro. 

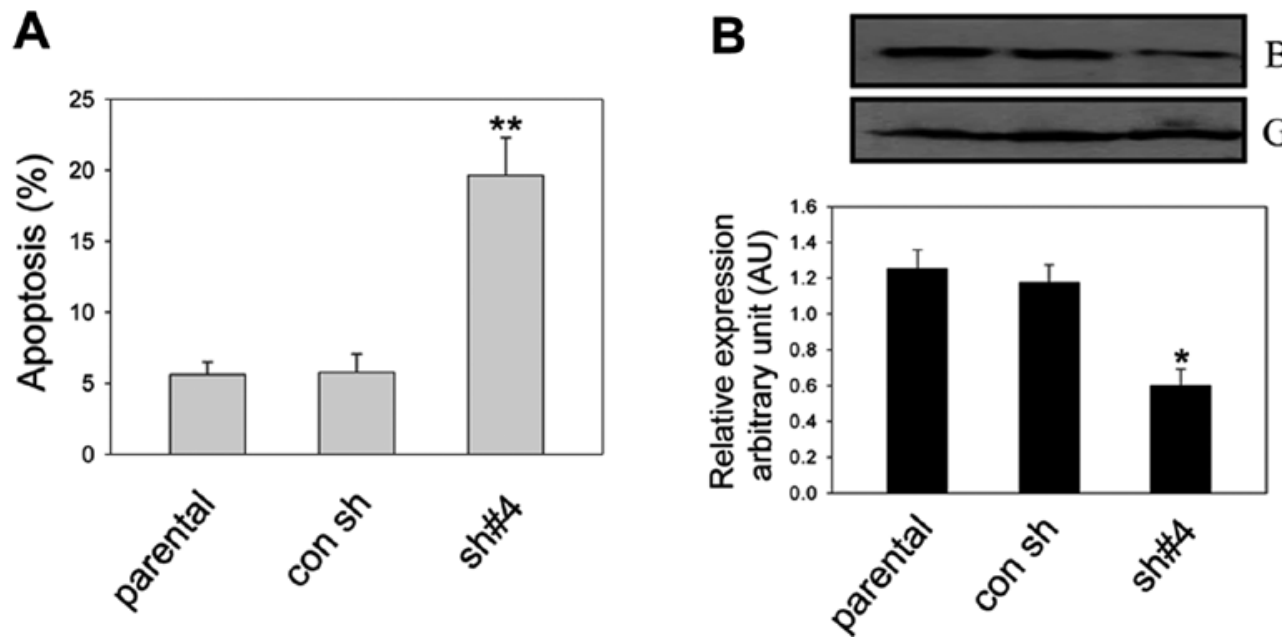

Figure 5. Lentiviral expression vector-mediated inhibition of VEGFR-3 promotes apoptosis in MKN45 cells. (A) MKN45 cells were collected $48 \mathrm{~h}$ after infection with con sh, sh\#4 lentivirus particles. Cell apoptosis was evaluated by the Annexin V binding assay. The percentage of cell apoptosis was determined as the ratio of apoptotic cell number to the total cell number. (B) Protein extraction from 3 different infected groups after $72 \mathrm{~h}$ and Western blot analysis assay was performed to determine the VEGFR-3 protein levels. Protein quantification was obtained by densitometric analysis of the protein band area. The protein product was quantified relative to the internal control GAPDH. ${ }^{*} \mathrm{P}<0.05 ;{ }^{* *} \mathrm{P}<0.01$. All of the experiments were repeated at least two times.

A

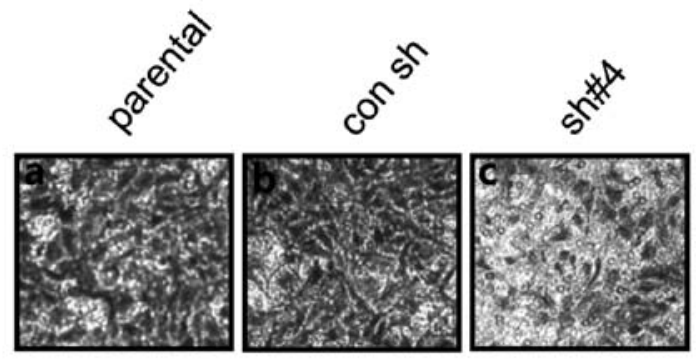

B

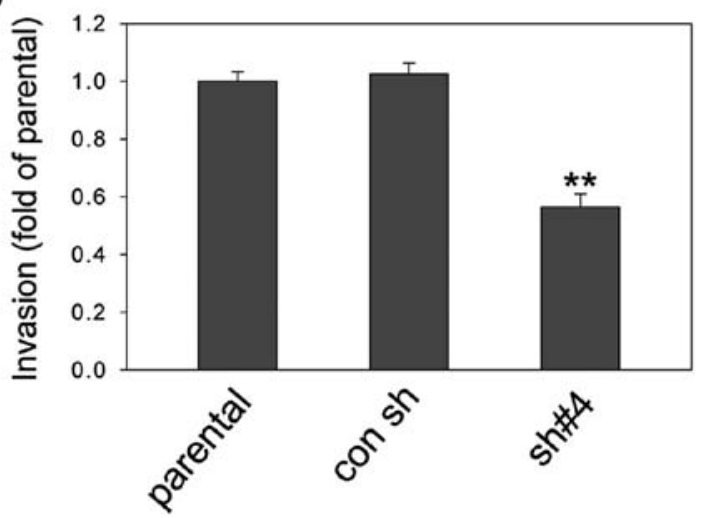

Figure 6. Lentiviral expression vector-mediated inhibition of VEGFR-3 suppresses cell invasion in the MKN45 cell line. (A) MKN45 cells were infected with con sh, sh\#4 lentivirus particles for $72 \mathrm{~h}$ and transferred to $8 \mu \mathrm{m}$ Transwell chambers coated with Matrigel. After $20 \mathrm{~h}$, the cells were scraped from the chamber and stained to check for invasion of cells to the lower surface. Representative cells that invaded through the Matrigel and the membrane were observed under a fluorescence microscope. (B) The invasion was quantified as described in Materials and methods. ${ }^{* *} \mathrm{P}<0.01$. All experiments were performed three times.

Effect of VEGFR-3 knockdown on the CNTN-1 expression in gastric cancer cell. Su et al have reported that the VEGF-C/ VEGFR-3 axis, through up-regulation of the downstream effector gene CNTN-1, promotes invasion and metastasis of lung adenocarcinoma cells (5). Therefore, we examined



Figure 7. VEGFR-3 knockdown down-regulates the level of CNTN-1 in the MKN45 cell line. Total-RNA was extracted from MKN45 cells $72 \mathrm{~h}$ after infection with con sh or sh\#4 lentivirus particles and the mRNA expression of CNTN-1 was detected by qPCR. ${ }^{*} \mathrm{P}<0.05$. All of the experiments were repeated at least three times.

whether VEGFR-3 signaling could regulate the expression of the downstream effector gene CNTN-1 in the gastric cancer cell line MKN45. qPCR analysis was performed to detect the mRNA expression of CNTN-1 in MKN45 cells infected with lentivirus particles for $72 \mathrm{~h}$. Compared to the other two groups, the mRNA level of CNTN-1 in the sh\#4 group was significantly decreased (Fig. 7).

\section{Discussion}

VEGF-C/VEGFR-3 is one of the most important angiogenesis axes and its role in tumor growth, metastasis and development have been well documented $(16,17,21,22,24,31)$. A study by Yonemura et al indicated that VEGF-C may induce the proliferation of lymphatic vessels in the stroma of primary gastric cancer via activation of VEGFR-3, expressed on the endothelial cells of lymphatic vessels. Under these circumstances, cancer cells could easily invade the lymphatic vessels (32). 
When VEGF-C was secreted by tumor cells, it could act as a paracrine factor to stimulate cell proliferation (33) and lymph node metastasis $(6,8,17-19)$. These findings led us to hypothesize that VEGF-C promotes tumor progression by binding directly to the VEGF receptor (VEGFR) in tumor cells $(13,14)$.

Previous studies reported that VEGFR inhibitor PTK787/ ZK222584 (28) or antibodies to VEGFR (29) could inhibit colon adenocarcinoma development. The use of liganddirected nanoparticles has shown promise in addressing other RNAi issues, such as cellular uptake, nonspecific immune stimulation, and limited stability $(9,16,34)$. Chemically or enzymatically synthesized siRNA oligos are costly and have been shown to have a relatively short half-life (25), with only transient inhibition of target genes. While many problems still exist on how to deliver the siRNA, repeated administration of siRNA may compensate for the reagent's short half-life.

RNAi has been used as a therapeutic tool (35). However, more attention should be paid on the delivery strategy which will make delivery of siRNA more efficient and easy. The present study highlights another technique: the use of shRNAbased, especially the shRNA-based recombinant lentiviral vectors to produce siRNA. This approach may offer more efficient siRNA delivery and gene silencing. In the present study, we constructed a recombinant lentiviral vector for RNAi targeting the VEGFR-3 gene, which was then packaged into lentivirus particles and used to infect the gastric cancer cell line MKN45, in order to observe its effect on VEGFR-3 gene expression, cell proliferation, cell cycle distribution, cell apoptosis and cell invasion. Results show that the sh\#4 group can effectively inhibit VEGFR-3 gene expression in the MKN45 cell line. Infected MKN45 cell proliferation and cell invasion in the sh\#4 group was lower and cell apoptosis was higher than that of the parental and con sh groups. The infected MKN45 cell division stopped at the G1 phase. Therefore, the lentiviral particles for RNAi, targeting the VEGFR-3 gene, can specifically suppress VEGFR-3 gene expression, regulate the cell cycle, promote apoptosis and inhibit proliferation of gastric cancer MKN45 cells. This is consistent with the results of previous studies with anti-VEGFR-3 blocking antibodies which show that inhibition of VEGFR-3 results in suppression of gastric cancer development (36). Simultaneously, our results can be explained by that VEGF-C/VEGFR-3 plays a critical role in the proliferation, migration and invasion of gastric carcinoma cells $(8,37)$.

These findings have prompted a novel strategy and targeting site for therapeutic treatment of gastric cancer. The results demonstrate that the VEGFR-3 shRNA lentivirus plasmid can effectively inhibit VEGFR-3 gene expression in the MKN45 cell line, which will be greatly helpful to further research. The fact that VEGFR-3 knockdown down-regulated the level of CNTN-1 in our study indicates that VEGFR-3 could positively regulate the expression of the downstream effector gene CNTN-1 in the gastric cancer cell line MKN45. However, there are limitations of this study. Further studies examining whether VEGFR-3, through up-regulation of CNTN-1, promotes proliferation, invasion and metastasis of gastric cancer are required.

In summary, the present study demonstrated for the first time that lentiviral vector-mediated RNA interference of VEGFR-3 successfully inhibited the expression of VEGFR-3 and the potent suppression of tumor progression was evident on proliferation, apoptosis, cell cycle transition and invasion of MKN45 cells. These findings suggest that the RNAi of the VEGFR-3 gene can be an effective therapeutic strategy for human gastric cancer in the future.

\section{Acknowledgements}

This study was supported by the Shanghai Natural Science Foundation (no. 07ZR14087).

\section{References}

1. Fidler IJ: The pathogenesis of cancer metastasis: the 'seed and soil' hypothesis revisited. Nat Rev Cancer 3: 453-458, 2003.

2. Suhardja A and Hoffman H: Role of growth factors and their receptors in proliferation of microvascular endothelial cells. Microsc Res Tech 60: 70-75, 2003.

3. Alitalo K and Carmeliet P: Molecular mechanisms of lymphangiogenesis in health and disease. Cancer Cell 1: 219-227, 2002.

4. Dumont DJ, Jussila L, Taipale J, et al: Cardiovascular failure in mouse embryos deficient in VEGF receptor-3. Science 282: 946-949, 1998.

5. Su JL, Yang PC, Shih JY, et al: The VEGF-C/Flt-4 axis promotes invasion and metastasis of cancer cells. Cancer Cell 9: 209-223, 2006.

6. Padera TP, Ancukiewicz M, Hoshida T, Fukumura D and Jain RK: Anti-VEGFR-3 therapy and lymph node metastasis [corrected]. Cancer Res 67: 5055-5056, 2007.

7. Han FH, Li HM, Zheng DH, He YL and Zhan WH: The effect of the expression of vascular endothelial growth factor (VEGF)-C and VEGF receptor-3 on the clinical outcome in patients with gastric carcinoma. Eur J Surg Oncol 36: 1172-1179, 2010.

8. Kitadai Y, Kodama M, Cho S, et al: Quantitative analysis of lymphangiogenic markers for predicting metastasis of human gastric carcinoma to lymph nodes. Int J Cancer 115: 388-392, 2005.

9. Pytowski B, Goldman J, Persaud K, et al: Complete and specific inhibition of adult lymphatic regeneration by a novel VEGFR-3 neutralizing antibody. J Natl Cancer Inst 97: 14-21, 2005.

10. Hirashima Y, Yamada Y, Matsubara J, et al: Impact of vascular endothelial growth factor receptor 1,2, and 3 expression on the outcome of patients with gastric cancer. Cancer Sci 100: 310-315, 2009.

11. Smith NR, Baker D, James NH, et al: Vascular endothelial growth factor receptors VEGFR-2 and VEGFR-3 are localized primarily to the vasculature in human primary solid cancers. Clin Cancer Res 16: 3548-3561, 2010.

12. Yang J, Wu HF, Qian LX, et al: Increased expressions of vascular endothelial growth factor (VEGF), VEGF-C and VEGF receptor-3 in prostate cancer tissue are associated with tumor progression. Asian J Androl 8: 169-175, 2006.

13. Valtola R, Salven $\mathrm{P}$, Heikkila $\mathrm{P}$, et al: VEGFR-3 and its ligand VEGF-C are associated with angiogenesis in breast cancer. Am J Pathol 154: 1381-1390, 1999.

14. Kubo H, Fujiwara T, Jussila L, et al: Involvement of vascular endothelial growth factor receptor-3 in maintenance of integrity of endothelial cell lining during tumor angiogenesis. Blood 96: 546-553, 2000

15. Laakkonen P, Waltari M, Holopainen T, et al: Vascular endothelial growth factor receptor 3 is involved in tumor angiogenesis and growth. Cancer Res 67: 593-599, 2007.

16. Partanen TA, Arola J, Saaristo A, et al: VEGF-C and VEGF-D expression in neuroendocrine cells and their receptor, VEGFR-3, in fenestrated blood vessels in human tissues. FASEB J 14: 2087-2096, 2000.

17. Mattila MM, Ruohola JK, Karpanen T, Jackson DG, Alitalo K and Harkonen PL: VEGF-C induced lymphangiogenesis is associated with lymph node metastasis in orthotopic MCF-7 tumors. Int J Cancer 98: 946-951, 2002.

18. Siriwardena BS, Kudo Y, Ogawa I, Udagama MN, Tilakaratne WM and Takata T: VEGF-C is associated with lymphatic status and invasion in oral cancer. J Clinical Pathol 61: 103-108, 2008.

19. He Y, Kozaki K, Karpanen T, et al: Suppression of tumor lymphangiogenesis and lymph node metastasis by blocking vascular endothelial growth factor receptor 3 signaling. J Natl Cancer Inst 94: 819-825, 2002.

20. Su JL, Yen CJ, Chen PS, et al: The role of the VEGF-C/VEGFR-3 axis in cancer progression. Br J Cancer 96: 541-545, 2007. 
21. Karpanen T, Egeblad M, Karkkainen MJ, et al: Vascular endothelial growth factor $\mathrm{C}$ promotes tumor lymphangiogenesis and intralymphatic tumor growth. Cancer Res 61: 1786-1790, 2001.

22. He XW, Yu X, Liu T, Yu SY and Chen DJ: Vector-based RNA interference against vascular endothelial growth factor-C inhibits tumor lymphangiogenesis and growth of colorectal cancer in vivo in mice. Chin Med J (Engl) 121: 439-444, 2008.

23. Skobe M, Hawighorst T, Jackson DG, et al: Induction of tumor lymphangiogenesis by VEGF-C promotes breast cancer metastasis. Nat Med 7: 192-198, 2001.

24. Stacker SA, Caesar C, Baldwin ME, et al: VEGF-D promotes the metastatic spread of tumor cells via the lymphatics. Nat Med 7: 186-191, 2001

25. Wang S, Liu H, Ren L, Pan Y and Zhang Y: Inhibiting colorectal carcinoma growth and metastasis by blocking the expression of VEGF using RNA interference. Neoplasia 10: 399-407, 2008.

26. Poyer F, Coquerel B, Pegahi R, et al: Secretion of MMP-2 and MMP-9 induced by VEGF autocrine loop correlates with clinical features in childhood acute lymphoblastic leukemia. Leuk Res 33: 407-417, 2009.

27. Masood R, Kundra A, Zhu S, et al: Malignant mesothelioma growth inhibition by agents that target the VEGF and VEGF-C autocrine loops. Int J Cancer 104: 603-610, 2003.

28. Hess C, Vuong V, Hegyi I, et al: Effect of VEGF receptor inhibitor PTK787/ZK222584 [correction of ZK222548] combined with ionizing radiation on endothelial cells and tumour growth. Br J Cancer 85: 2010-2016, 2001.

29. Shaheen RM, Ahmad SA, Liu W, et al: Inhibited growth of colon cancer carcinomatosis by antibodies to vascular endothelial and epidermal growth factor receptors. Br J Cancer 85: 584-589, 2001
30. Mineva I, Stamenova M, Gartner W and Wagner L: Expression of the small heat shock protein alphaB-crystallin in term human placenta. Am J Reprod Immunol 60: 584-589, 2008.

31. Ishikawa M, Kitayama J, Kazama S and Nagawa H: Expression of vascular endothelial growth factor C and D (VEGF-C and -D) is an important risk factor for lymphatic metastasis in undifferentiated early gastric carcinoma. Jpn J Clin Oncol 33: 21-27, 2003.

32. Yonemura Y, Fushida S, Bando E, et al: Lymphangiogenesis and the vascular endothelial growth factor receptor (VEGFR)-3 in gastric cancer. Eur J Cancer 37: 918-923, 2001.

33. He Y, Rajantie I, Pajusola K, et al: Vascular endothelial cell growth factor receptor 3-mediated activation of lymphatic endothelium is crucial for tumor cell entry and spread via lymphatic vessels. Cancer Res 65: 4739-4746, 2005.

34. Shida A, Fujioka S, Kobayashi K, et al: Expression of vascular endothelial growth factor (VEGF)-C and -D in gastric carcinoma. Int J Clin Oncol 11: 38-43, 2006.

35. Elbashir SM, Harborth J, Weber K and Tuschl T: Analysis of gene function in somatic mammalian cells using small interfering RNAs. Methods 26: 199-213, 2002.

36. Shimizu K, Kubo H, Yamaguchi K, et al: Suppression of VEGFR-3 signaling inhibits lymph node metastasis in gastric cancer. Cancer Sci 95: 328-333, 2004

37. Pepper MS, Tille JC, Nisato R and Skobe M: Lymphangiogenesis and tumor metastasis. Cell Tissue Res 314: 167-177, 2003. 